\title{
A snapshot of intellectual disabilities in Lao PDR: Challenges for the development of services
}

\section{Authors}

Dr Stian H Thoresen, Curtin University, Australia

Dr Angela Fielding, Curtin University Australia

Dr Sue Gillieatt, Curtin University, Australia

Dr Barbara Blundell, Curtin University, Australia

Dr Liem Nguyen, Curtin University, Australia

\section{Corresponding Author}

Stian H Thoresen, School of Occupational Therapy and Social Work, Curtin University, GPO Box U 1987, Perth Western Australia 6845, Australia

Email: s.thoresen@curtin.edu.au

\section{Funding}

The authors disclosed receipt of the following financial support for the research, authorship, and publication of this article: This research work was financially supported by an Australian Development Research Awards Scheme grant by Australian Aid, Department of Foreign Affairs and Trade, Australian Government, Grant Agreement 66447.

\section{Acknowledgement}

The authors acknowledge the substantial contributions towards the project by numerous stakeholders, particularly the National Committee for Disabled and Elderly at the Ministry of Labour and Social Welfare, the Lao Disabled People's Association (LDPA), and the Association for Autism (AfA) in Lao PDR. A Reference Group consisting of key stakeholders for persons with disabilities in 
Lao PDR has guided the larger study that this article draws on and the authors acknowledge the members' valuable and substantial contributions.

\section{Abstract}

Lao People's Democratic Republic (PDR) has experienced significant economic growth in the last two decades[AQ1]. While access to education, health, and employment for people with disabilities has also improved markedly, people with intellectual disabilities are still among the most vulnerable in society. This article presents findings from a screening survey of 2469 participants, a focus group discussion with key stakeholders in intellectual disabilities, and a case study of the Association for Autism. The screening survey suggests $1.9 \%$ of working age participants (18-59) and $0.7 \%$ of children (6-17) have intellectual disabilities. The qualitative data illustrate poor understanding and stigmatization of intellectual disabilities, lack of services, and development of a parent association to improve the life prospects for children with autism and intellectual disabilities. People with intellectual disabilities in Lao PDR remain particularly vulnerable. Parents and other disability advocates are raising awareness and demonstrating the effectiveness of specialized services.

Keywords: access to services, autism spectrum disorders, disability-inclusive, development, Lao PDR, parent association 


\section{A snapshot of intellectual disabilities in Lao PDR: Challenges for the development of services}

\section{Introduction}

This article presents selected information collected as part of a larger mixed-methods study investigating access to social and economic services for people with disabilities in Lao People's Democratic Republic (Lao PDR). In additions to the findings from a structured scoping review, this article presents findings from a screening survey, a focus group discussion, and a case study pertinent to the development of services for people with intellectual disabilities. Lao PDR is a developing country in Southeast Asia where disabilities have been most recently understood within a war context. While the conceptualisation of and engagement with disabilities in Lao PDR has developed significantly over the past decade, there has been a traditional emphasis on physical disabilities caused by unexploded ordnance (UXO) and remnants from war (Thoresen et al., 2014). UXOs from the Viet Nam, or Indochina, War are still killing and disabling hundreds of Laotians annually (Durham et al., 2013; Durham and Hoy, 2013; Morikawa et al., 1998). This has meant that other types of disabilities, including intellectual, receive far less attention.

Lao PDR has a population of about 6.5 million people belonging to many ethnic groups (Lao Statistics Bureau, 2015), and is classified by the United Nations (2016) as a Least Developed Country (LDC). Lao PDR has been an LDC since classification began in 1971; a compound assessment across the criteria of 'low income' mainly based on gross national income; 'human assets weakness' related to nutrition, health, and education; and 'economic vulnerability' related to natural and trade-related shocks (United Nations Development Programme, 2012). However, economic growth was tracking at an average of $8 \%$ in 2012 and Lao PDR is the only LDC country aspiring to graduate from LDC status by 2020 (Cooper, 2014). 
The United Nations Convention on the Rights of Persons with Disabilities (UNCRPD) (United Nations General Assembly, 2006) has provided important impetus to advocate for enhancing the rights and full citizenship of people with disabilities, and has in many instances facilitated the development and implementation of laws, policies, and practices to provide improved support and services for people with disabilities. These developments have been supported by improved disability-inclusive international development frameworks, including explicit targets for people with disabilities in the Sustainable Development Goals (SDGs) (United Nations, 2015), that were absent from the Millennium Development Goals (MDGs) (Thomas, 2005).

Other international frameworks focusing on disabilities have been developing fast, especially in the Asia-Pacific region, and these have enabled recognition of the importance of rights for people with disabilities. The increased emphasis on disability-inclusive development across the region and internationally (Australian Government, 2008; Commonwealth of Australia, 2015; United Nations Economic and Social Commission for Asia and the Pacific, 2012; United Nations Economic and Social Commission for Asia and the Pacific, 2014) has enabled substantial progress over the past decade. In the Asia-Pacific, the third Decade of Persons with Disabilities (2013-2022) and the Incheon Strategy (United Nations Economic and Social Commission for Asia and the Pacific, 2012) have promoted disability-inclusive development and have established a framework to monitor the progress of implementing the UNCRPD in the region. The Incheon Strategy includes 10 goals covering social, political, and economic participation and inclusion; accessibility; education; gender equality; disaster risk reduction; data on disabilities; and regional cooperation. Specific indicators and reporting requirements have also been developed (United Nations Economic and Social Commission for Asia and the Pacific, 2014). These international frameworks have established a foundation for disabled people's organisations (DPOs) and other advocates to lobby governments to improve the rights and access to services for people with disabilities. For countries in the region, this provides important leverage to ensure that people with disabilities, as some of the most disadvantaged in society, are included in development strategies. 
While understandings about disabilities in Lao PDR are developing, there is concern about the social and economic inclusion of people with disabilities, particularly people with intellectual disabilities. There is apprehension that the most vulnerable, people with severe and profound disabilities, are not included to the same degree, as has been identified more broadly (World Health Organization 2007). Targets and key performance indicators are often only applied to people with disabilities in the broadest sense, and do not distinguish between outcomes for people with mild or moderate disabilities and those with profound and severe disabilities, who may require more resources to reach similar outcomes. People with intellectual disabilities, particularly those with higher support needs, are at high risk of exclusion, particularly if they have communication difficulties and rely on others to advocate on their behalf. In low-income countries, this translates to even greater isolation and disadvantage across the lifespan (Cohen et al., 2015).

This article presents preliminary findings from a larger study investigating access to social and economic services for people with disabilities in Lao PDR. Drawing on the participatory, mixedmethods study, this article presents findings related to prevalence, and availability of and access to services for people with intellectual disabilities, as perceived by key stakeholders such as government ministries or agencies, service providers, and DPOs, including parent-led advocacy organisations. Specifically, this article outlines what is known about people with intellectual disabilities in Lao PDR, particularly intellectual disabilities and autism spectrum disorders (hereafter referred to as autism), and explores health and education services, paying particular attention to accessibility, availability, affordability, and acceptability, the 4As (Jacobs et al., 2012). It also includes a description of a parent-led service delivery response in the case of intellectual disabilities and autism. 


\section{Background}

Intellectual disabilities in Lao PDR

There is a dearth of information with reference to intellectual disabilities in Lao PDR. However, recent initiatives to collect disability-statistics using international guidelines are facilitating greater understanding of intellectual disabilities. While only preliminary findings are available from the latest Lao Population and Housing Census carried out in 2015 (Lao Statistics Bureau, 2015), it did utilise the Washington Group's Short Set of Questions on Disability (Mandans et al., 2011). The Washington Group questions explore the degree of difficulty with seeing, hearing, walking, remembering or concentrating, self-care, and communicating, shifting the emphasis to functioning rather than to individual deficits. While by no means exclusive, level of difficulty remembering or concentrating can indicate intellectual disabilities for young and working-aged people. However, the operationalisation of intellectual disabilities in this way excludes other common indicators or comorbid challenges related to intellectual disabilities, such as communication difficulties.

In contrast, the preceding 2005 census asked: 'is there any disabled person in this household?' (Steering Committee for Census of Population and Housing, 2004). This direct question indicated that $1.3 \%$ of the Lao population have disabilities (Messerli et al., 2008). This was followed with a question around specifying type of disabilities according to five classifications: 'visually impaired' (17\%), 'deaf or dumb' (27\%), 'arm or leg handicapped' (39\%), 'multiple disabilities' (7\%), and 'other disabilities' (10\%). The census also sought to identify the cause of disabilities according to six classifications: 'at birth' (39\%), 'war' (11\%), 'accident' (16\%), 'drugs' (1\%), 'disease' (28\%), and 'other' (5\%) (Messerli et al., 2008). People with intellectual disabilities could have been categorised within either the 'multiple disabilities' or 'other disabilities' classifications of the 2005 census. 
The limited information available on intellectual disabilities from the 2005 census has partly contributed to the current limited understandings of both intellectual disabilities and other 'nonvisible' disabilities such as mental health distress or mental illness; both generally not perceived as disabilities in Lao PDR (Thoresen et al., 2014). However, disability-advocates are challenging the traditional deficit approach to disabilities, including any categorising which is based on traditional classifications of impairment. Improving disability-data will support these efforts.

\section{Scoping review}

In the first half of 2016, the authors conducted an English language scoping review of formal and grey literature about the global prevalence of intellectual disabilities as well as what is known about intellectual disabilities in the Association of Southeast Asian Nations (ASEAN) and the Greater Mekong Sub-region (GMS). Emphasis was placed on health and education services in the GMS and ASEAN countries from 2000 onwards. GMS countries include Cambodia, the People's Republic of China (specifically Yunnan Province and Guangxi Zhuang Autonomous Region, but specifically excludes the Special Administrative Regions of Hong Kong and Macau), Lao PDR, Myanmar, Thailand, and Viet Nam, while ASEAN excludes China but includes the remainder of GMS countries with the addition of Brunei Darussalam, Indonesia, Malaysia, Philippines, and Singapore. Keywords for this review included the geographical regions AND intellectual disabilities OR developmental delay OR cerebral palsy OR Down syndrome OR autism. Final inclusion criteria were intellectual disabilities (independent of comorbidities) within at least one of the 11 GMS or ASEAN countries published in 2000 or later.

Multiple database searches were conducted to identify publications for inclusion. The titles and abstracts of the 51 identified articles were uploaded into Covidence (www.covidence.org), which was then used to organise screening of all titles and abstracts by author pairs. Conflicts were resolved by discussion between two of the reviewers. Full-text articles were available for 17 of the 
22 publications identified as potentially relevant, and these were reviewed for inclusion. After full review, 12 of these articles were found to contain relevant material for inclusion. Additional handsearches identified further relevant publications, particularly related to intellectual disabilities globally as well as pertinent studies from not only GMS or ASEAN countries but also other countries in the region like Taiwan, Western China, and Hong Kong.

In 2006, Harris reported the global prevalence of intellectual disabilities as varying between 1\% and 3\% (Harris, 2006). In 2011, Maulik and colleagues produced a meta-analysis of 52 studies published over the last 30 years, across the lifespan in high, middle and low-income countries, and found a general prevalence of $1.0 \%$. However, the prevalence in the low-income countries studies included in the meta-analysis was higher at 1.6\% (Maulik et al., 2011). Scior et al. (2015) also report an estimate of between $1 \%$ and $3 \%$ within the general population, although intellectual disabilities are regarded as more common in low-resource countries where there is a greater dependence on parents, parents' associations and non-government organisations (NGOs) to advocate and even provide education services for people with disabilities (Scior et al., 2015). According to Scior et al. (2015), despite the UNCRPD, adults and children with intellectual disabilities remain largely invisible. In 2011, Elsabbagh et al. (2012) conducted the first systematic review methodology of epidemiological surveys across the world about the prevalence of autism and other pervasive developmental disorders. They found the median of prevalence estimates of autism was $0.6 \%$ with no surveys for low-income countries available for review.

There is scarce information available regarding the prevalence of intellectual disabilities across the GMS or ASEAN. However, the Thai 2012 Disability Survey identified 68,136 persons with intellectual disabilities and 42,890 persons with learning disability in the Kingdom (National Statistical Office and Ministry of Information and Communication Technology, 2014). Acknowledging that it is often difficult to distinguish between intellectual and learning disabilities, these figures would suggest a combined prevalence of about $0.2 \%$ (calculated based on population of $68,007,361$ 
persons). For Vietnam, the 2009 Population and Housing Census suggests that the proportion of people aged 5 years or older with cognition limitations is $3.5 \%$ with 'at least some difficulty', $0.8 \%$ for 'at least a lot of difficulty', and $0.2 \%$ for 'unable/cannot do at all' (UNFPA, 2011).

The causes of intellectual disabilities are complex and often undetermined. Given the absence of studies accounting for intellectual disabilities in Lao PDR, it would be speculative to propose its major causes. However, to the authors' knowledge, there is no evidence to suggest that the cause of intellectual disabilities differs from elsewhere across the world. In Australia, for example, about $54 \%$ of cases of intellectual disabilities are related to genetic disorders; about $17 \%$ of cases relate to disease, illness, or hereditary disorders; about $8 \%$ of cases are related to other causes; while in $22 \%$ of cases, the cause is unknown (AlHW, 2008). However, Emerson (2007) has established a causal effect between poverty and intellectual disabilities, illustrating the importance of socioeconomic and other environmental factors. Thus, further research into the causes and comorbidities of intellectual disabilities in Lao PDR may be required to facilitate the development of more targeted education and health services.

\section{Access to education and health services}

Across the region, details about available, affordable and acceptable education and health services are scant, including those related to community-based rehabilitation for children and adults with intellectual disabilities. Services are often hospital-based, or if community-based, they are NGOinitiated and driven, and can be fragmented (Amar-Singh, 2008; Robertson et al., 2012; Poon, 2015; Kuipers and Maratmo, 2011). An absence of well-trained health professionals able to provide support and interventions for people with intellectual disabilities means families often step in to deliver services, making parent-led interventions common-place (Einfeld et al., 2012). Success in putting inclusive education strategies in place for children with disabilities is seen as being influenced by attitudes, with the development of positive attitudes by key stakeholders the most 
decisive factor for successful inclusion (Deng and Holdsworth, 2007). In the past, teachers and head teachers in Western China expressed a key concern that poor boys and girls were not attending school; the implication being that the attendance of students with disabilities was somehow less important given the larger number of students not attending from lower socio-economic backgrounds. In addition, the inclusion of 'slow' students would mean a lower academic average for the whole class, placing the teacher in a disadvantageous position in comparison with other teachers (Deng and Holdsworth, 2007: 515).

It has been proposed that in China, autism is often not recognised as a disability, properly diagnosed, or catered for in schools (Deng and Holdsworth, 2007). Other barriers to inclusion in mainstream classrooms include large class sizes and a lack of teacher training and experience in working with children with autism (Peters and Forlin, 2011). An article by Nazri and Ismail (2015) about educational building facilities for children with autism in Malaysia proposes that the physical learning environment is important. The quality and appropriateness of the learning environment affects outcomes, so that design of inclusive learning spaces is vital.

Kwok and Chui (2008) surveyed mental health care for adults with intellectual disabilities in Asia and service provision around intellectual disabilities more broadly, and found that the range and type of services available for people with intellectual disabilities varied significantly. China, Thailand and the Philippines were reported as using normalisation and community integration as primary objectives in service provision for people with intellectual disabilities (Kwok and Chui, 2008). Viet Nam was reported to provide no formal services for adults with intellectual disabilities in the areas of accommodation, training or employment (Kwok and Chui, 2008). The absence of studies focusing on intellectual disabilities in Lao PDR is noteworthy. 


\section{Methods}

This article draws on findings from a screening survey, a focus group discussion, and an illustrative case study. The study was granted approval from the Human Research Ethics Committee at the authors' university in late 2014 (approval number HR 217/2014).

Screening survey on prevalence

A screening survey was carried out in late 2015 to identify people with and without disabilities based on functional capacity according to the International Classification of Functioning, Disability, and Health (ICF). The selected study sites were 12 villages from six districts in Sayaboury, Vientiane, and Savannakhet provinces (two districts in each province and two villages in each district). The selection of study sites was made in consultation with the project's reference group, comprised of key disability-stakeholders in Lao PDR from DPOs, key government ministries and agencies, international disability-NGOs, and international development agencies. The final selection of districts and villages in the selected provinces was made in consultation with Provincial and District leaders with one urban and one rural village from each district.

The screening survey targeted the 'de facto' population, operationalised as individuals who had spent the previous night in the village. This differs from the prevailing methodology across the region, as most household surveys seek information related to all members listed on the household registers and usually obtains this from the household head on behalf of all household members. While there are exceptions to the approach described above, particularly regarding gender-specific questions (for example, related to domestic violence, maternal and sexual health), the present study did not accept proxy-only responses, with the exception of parents or guardians responding on behalf of children. This approach was adopted to limit potential bias that proxy respondents 
introduce, but family members were encouraged to support participants as appropriate (for example participants with intellectual or other cognitive disabilities).

The study drew on the Washington Group's Short Set of Questions on Disability (Mandans et al., 2011) to identify people with and without disabilities. These questions investigate the level of difficulty respondents have across six domains: seeing, hearing, walking, remembering or concentrating, self-care, and communicating, and have been recommended as a standard inclusion in censuses to identify persons with disabilities (Mandans et al., 2011). However, it must be noted that the Washington Group questions were developed for adults and the Group is currently working with the United Nations Children's Fund to develop and validate an instrument for children aged 217 (Washington Group, 2014). Recognising that the Washington Group questions may not be suitable for very young children, this study only included participants born before 2010 (that is, excluding children aged five years or younger). In total 2,469 individuals from 939 households responded to the screening survey.

Focus group on intellectual disabilities

Given the limited information available related to intellectual disabilities in Lao PDR, a focus group was held in early 2016 with key stakeholders in general disabilities as well as intellectual disabilities; six representatives from key government agencies, DPOs, and service providers. The purpose of the group was to specifically discuss the experiences of families and services in relation to intellectual disabilities. Participants represented health and rehabilitation, education, DPOs including two parent organisations and had knowledge of general disabilities and specific programs for intellectual disabilities. Three participants contributed their personal experiences as parents in addition to their roles as service providers. The focus group discussion was facilitated by one of the researchers and supported by an interpreter. It followed an informal format, was exploratory in nature, and centred around five main questions or objectives: 
1. Discussion of facts, myths, and beliefs related to disabilities generally and intellectual disabilities specifically.

2. Meaning or understanding of intellectual disabilities.

3. Available services in Lao PDR for people with intellectual disabilities.

4. How people access services.

5. What facilitates participation in education, employment, and social activities?

The focus group was video- and audio-recorded. A summary of the group discussion was compiled and supplemented by the researcher's notes. The information was verified by clarifying interpretations and meanings with the participants and the interpreter, checking several sources for accuracy, and then analysed thematically (Miles and Huberman, 1994; Hall and Kramer, 2009).

\section{Case study of parental group of children with autism and intellectual disabilities}

Given the larger study sought to understand and describe the experiences of people with disabilities and to identify barriers and facilitators to participation in and access to health, education, employment and social activities, case studies were also identified to provide depth of information. This additional data provided a good counterbalance to the quantitative survey, and highlighted specific points and practices. The case study presented in this article was developed around the Association for Autism (AfA) which was established by parents of children with autism and intellectual disabilities.

The case study was constructed using a variety of sources over a two-year period; 2014 to 2016. The majority of the information was gathered during four visits to the Centre for Autism in Vientiane, with additional information, observations, and reflections collected during public events, a school visit and other meetings related to the larger study that included AfA representatives. The data sources used were: 
- Interviews with the stakeholders about facilitators and barriers to inclusive practices.

- Interviews with persons and/or parents of persons with and without disabilities about their lived experience.

- Participant observation in organisations and community to learn about different practices.

- Noting physical artefacts such as equipment, assistive devices, open access buildings, public transport, and the built environment including pavements and city infrastructure.

- Review of existing literature and documents provided by the stakeholders.

Data analysis followed similar qualitative methods that were used for the focus group and the AfA was consulted in the development of this article.

\section{Findings}

\section{Screening survey on prevalence}

With the overarching purpose of the screening survey being to identify people with and without disabilities based on the ICF framework, the use of the Washington Group's Short Set of Questions on Disability meant the researchers were unable to report the proportion of study participants with diagnosed intellectual disabilities. The findings from the focus group discussion and the case study also confirmed a limited capacity to diagnose intellectual disabilities in Lao PDR. The screening survey included the question: 'Do you have difficulty remembering or concentrating?' with the following four closed response categories: 'No, no difficulty'; 'Yes, some difficulty'; 'Yes, a lot of difficulty'; and, 'Cannot do at all'. This enabled postulation as to the prevalence of intellectual disabilities among the study participants, while acknowledging the challenges to operationalising intellectual disabilities, as noted in the background section. 
The responses to the Washington Group question about difficulties remembering or concentrating are presented in Table 1 according to three age groups: young people (6-17), workingaged adults (18-59), and older persons (60 years or older). The recommended procedure to identify people with disabilities is to classify respondents who report 'no difficulty' or 'some difficulty' as people without disabilities and respondents reporting 'a lot of difficulty' or 'cannot do at all' as people with disabilities (Loeb, 2012). Among participants of the screening survey, $0.7 \%$ of the young people reported 'a lot of difficulty' remembering or concentrating (none reported 'cannot do at all') while $1.6 \%$ and $0.2 \%$ of working-aged adults replied a 'lot of difficulty' or 'cannot do at all'. Recognising the challenges of distinguishing cognitive impairments associated with ageing from the cognitive impairments associated with developmental disabilities, the $10.2 \%$ of older adults who replied a 'lot of difficulty' (none reported 'cannot do at all') is noteworthy. This data was not included for this analysis since it would very likely incorrectly inflate the prevalence of intellectual disabilities. Therefore, a reasonable estimate of the prevalence of intellectual disabilities among the screening survey participants is $0.7 \%$ for young people and $1.9 \%$ for working-aged adults, or $1.6 \%$ for all participants aged 6-59 years of age. It is important to note that this estimate does not reflect diagnosed conditions. 
Table 1: Proxy indicator of prevalence of intellectual disabilities in survey sample

\section{Difficulty remembering or concentrating}

\begin{tabular}{lccccc} 
Age group & None & Some & A lot & Cannot do & All \\
& $\mathbf{n}(\%)$ & $\mathbf{n}(\%)$ & $\mathbf{n}(\%)$ & $\mathbf{n}(\%)$ & $\mathbf{n}(\%)$ \\
\hline $6-17^{(\mathrm{a})}$ & $530(96.7)$ & $14(2.6)$ & $4(0.7)$ & - & $548(100.0)$ \\
$18-59$ & $1,432(88.4)$ & $158(9.8)$ & $26(1.6)$ & $4(0.2)$ & $1,620(100.0)$ \\
$60+$ & $191(64.7)$ & $74(25.1)$ & $30(10.2)$ & - & $295(100.0)$ \\
\hline Total & $2,153(87.4)$ & $246(10.0)$ & $60(2.4)$ & $4(0.2)$ & $2,463(100.0)$
\end{tabular}

Notes Percentages have been rounded.

A dash (-) represents a true zero.

The Table includes information from 2,463 of the 2,469 participants due to missing data.

(a) The screening survey was carried out in December 2015 and children born in 2010 or later were excluded. It is possible that the sample includes some children age 5 years old if their birthday was in late December 2009.

Focus group on intellectual disabilities

Focus group participants confirmed very few services available for children with intellectual disabilities, and few job opportunities for adults. In spite of an inclusive education approach, minimal funding is provided for facilities or for specialised training on intellectual and disabilities for teachers, physiotherapists, or occupational therapists. There is also little coordination of the available services.

One parent outlined her experience with her son, who is now 20 years old. He spent his first two years in hospital, and then she managed him on her own at home for many years. In recent times, she has had some support such that he can now bathe himself. He has grown to adult size, and demands food all the time. He is getting very fat, but has to be given what he wants to keep him calm. She now helps raise awareness for other parents. One of the government service providers 
was in the process of establishing an early intervention program for children with significant developmental delay and autism, with staff being sent to Thailand for short-term training courses.

The focus group also included considerable discussion around the diversity of intellectual disabilities: co-morbidities, range of support needs for specific conditions, and poor understanding of intellectual disabilities more broadly in society. In addition, the challenges around definitions and diagnosis also reflected the lack of in-country expertise. Participants expressed particular concern that unless there was a visible co-existing condition, such as cerebral palsy or Down syndrome, children with intellectual disabilities were generally not diagnosed, leaving them without access to intervention, support, or treatment services. There is no substantial capacity in Lao PDR to diagnose intellectual disabilities, including syndrome-specific conditions. In addition, the policy environment for developmental disabilities is generally poor. The majority of support provided is for acquired disabilities, which largely excludes people with intellectual disabilities. While the few agencies catering for people with intellectual disabilities send staff for training in neighbouring countries, such as Thailand and Viet Nam, proper diagnosis of intellectual disabilities and related conditions also requires access to services in these countries, particularly Thailand. Only affluent families are able to cover these associated costs. In addition, the social and cultural stigmatisation of persons with disabilities and their families, particularly intellectual and psychosocial disabilities, is a major barrier to the provision of adequate support and services. Representatives of government health and education services explained the struggle to develop services within limited budget allocations. This compounds the challenges to social and community integration for people with disabilities in Lao PDR. People with intellectual disabilities, who rely on others to advocate on their behalf, are even more vulnerable. 
Case study of parental group of children with autism and intellectual disabilities

In 2005, a group of parents concerned about the development and behaviours of their children and unable to achieve a diagnosis within Lao PDR formed the Association (AfA). This case study illustrates what parents have been able to achieve through lobbying and taking on the role of developing new services for their children. Their experience was that intellectual disabilities, autism, and mental health problems are given very low priority by the Government. This may reflect some superstitions, for example, that the mother is to blame if children are born with disabilities, possibly linked with religious associations to karma and sins in previous lives. Additionally, there have been some legal impediments, such as the law on social security, which excludes people who have disabilities from birth. Only recently, the law on education was amended to include the right of access to education. However, it only refers to physical conditions and could be interpreted as excluding people with vision, hearing, intellectual, or psychosocial disabilities.

AfA parents have taken their children to Thailand for a diagnosis of autism, provided information and support to other parents, and have been working on providing suitable education services for their children. The AfA opened the Centre for Autism Vientiane in June 2009 in a house which is owned by one family, and now provides early intervention, and education support for more than 40 children with autism and intellectual disabilities. It is expensive to provide the services, partly due to the high support needs of the children requiring a high staff-to-child ratio. The AfA continues to apply for funding from a variety of international donors and has had some success.

AfA parents have lobbied the Ministry of Education and Sports (MOES) to train special education teachers and also sent their own staff to Thailand for training, hosted international child psychologists, and designed facilities based on this new information. While the Inclusive Education Centre (IEC) is making changes within the education sector, the challenges to bringing about systemic improvement are significant. The IEC sits within the Department of Primary and PrePrimary Education within the MOES. Frustrated at the slow response of government and needing 
support while their children were growing, the parents worked with a local primary school to have their children included with the support of their own staff. Even when the Ministry supported this initiative, there were significant delays at the District level.

Now that some of the children are entering their teens, the parents are starting to look at plans for post-school activities. They say that what they have achieved has only been possible because they were initially a relatively wealthy group of parents, who were willing to work together to seek the support they needed for their children from other countries. Since then, they have raised funds to provide scholarships to fund training and the education fees for 16 of the 38 children. An ongoing challenge is that after the AfA has paid for their staff to attend training, some have left to take up equivalent positions in government services. This means that investing in the training of AfA staff is an ongoing commitment.

The AfA now provides awareness-raising workshops in other parts of the country, and provides specialist training in Vientiane with international experts. As the initial AfA cohort of children grow up, the range of challenges the Association has to address will broaden to include vocational education and training, employment opportunities, broader social and community integration, and living arrangements.

\section{Discussion}

\section{Prevalence of intellectual disabilities}

Findings from the screening survey suggest that the prevalence of intellectual disabilities is about $1.9 \%$ for working-age adults or $1.6 \%$ for people aged between 6 and 59 years of age in the study sites. This is higher than the $1.3 \%$ prevalence rate for all types of disabilities in Lao PDR based on the 2005 Population and Housing Census (Messerli et al., 2008). This may reflect the different 
conceptualisations or approaches to operationalising and identifying disabilities. Differences in the quality of enumerators, level of supervision, implementation of surveys versus censuses may also contribute to this. It has also been noted that the prevalence of disabilities is usually higher in surveys compared to censuses, usually accounted for by differences in the types of questions (Mont, 2007).

Recognising that disability is along a continuum (World Health Organization, 2001), methodological decisions regarding cut-off points to determine the population with and without disabilities have obvious repercussions. The World Report on Disability suggests that $8.0 \%$ of Laotians have disabilities, which reflects the findings from the World Health Survey carried out between 2002 and 2004 (World Health Organization and The World Bank, 2011). Using a narrow and expanded conceptualisation of disabilities among persons aged 18 to 65 years of age, Mitra et al. (2011) conducted a secondary analysis of the data available from the World Health Survey for Lao PDR and concluded that the proportion of people with disabilities in Lao PDR was 3.1\% and $12.7 \%$ respectively. Using the expanded conceptualisation, $1.3 \%$ of the working-age population had difficulties 'concentrating/remembering things'. While the estimate by Mitra et al. (2011) is lower than the $1.9 \%$ of participants identified as having difficulties remembering or concentrating in the screening survey, both estimates are within the range of the 1-3\% global prevalence of intellectual disabilities suggested by Harris (2006). The slight difference in operationalisation of working-age between the screening survey and the World Health Survey should also be noted.

Lack of services for people with intellectual disabilities

Recognising that there are substantial challenges in facilitating the inclusion of people with intellectual disabilities into society in Lao PDR, particularly in normative socioeconomic roles, there is growing acknowledgement of intellectual disabilities across the country. The 'Decree on Persons with Disabilities' (Lao People's Democratic Republic, 2014) provides the first national definition of 
disabilities in Lao PDR. Although this definition takes a deficit approach, emphasising 'anomal[ies] or defects', intellectual disabilities are firmly encapsulated within this framework and work is ongoing to progress the Decree into Law and Policy.

A challenge in improving services for people with intellectual disabilities in Lao PDR is the wide range of support needs among people with intellectual disabilities. To maximise the limited resources available, equity groups are often clustered together. For example, the inclusive education programs and strategies that have run successfully in Lao PDR from the early 1990s are aimed at improving access to education for girls, ethnic minorities, and children with disabilities. Children with mild disabilities have reaped substantial benefits from these initiatives, and concern remains over limited access for children with severe and profound disabilities (Grimes, 2009; Grimes et al., 2011).

The AfA provides a case study of parental leadership for people with autism and intellectual disabilities in Lao PDR. It is a reminder of how services have historically developed across the world through parent activities due to the lack of, or inappropriateness, of government-provided services. As illustrated by the case study, there is a lack of specialists in intellectual disabilities in Lao PDR. However, by investing in up-skilling their staff, the AfA has been able to expand their services. Nevertheless, the focus group discussion, illustrated the likely limited availability of other services for people with intellectual disabilities in Lao PDR. While Chou et al. (2008) found that services in Taiwan were often underutilised due to lack of awareness about what was available, the situation in Lao PDR is more likely to reflect a general lack of available services. This was also demonstrated by the fact that a majority of focus group participants represented broad disabilities or service organisations, rather than intellectual disabilities organisations, although they may have included programs for people with intellectual disabilities. 
Studies have proposed that early intervention for children with developmental disabilities is important in promoting optimal development and in the reduction of the impact of the disabilities (Juneja et al., 2012a). While this is a standard approach in developed countries, the requisite legislation and funding is often lacking or improperly implemented in developing countries (Juneja et al., 2012a). Lack of available services may mean that parents are very involved in providing and assisting interventions, and it has been proposed that parent-based behavioural interventions may be a solution to lack of services available in low resource settings (Juneja et al., 2012b). Parents and other family members are often required to provide substantial leadership to exert change and provide good outcomes for people with intellectual disabilities (Cocks et al., 2016).

Effective early intervention relies on early diagnosis. Both the focus group and case study identified that the lack of diagnostic expertise in Lao PDR has meant families often have to travel to neighbouring countries to obtain a diagnosis for their child. This is unattainable for families from low socioeconomic backgrounds, arguably the majority of Laotians. The AfA is an example of how parents, in this case as a parent association, can band together to redress the inadequacies or complete lack of public services for children with intellectual disabilities.

There are countless examples of services for people with disabilities being developed by parents across the world to counter the lack of, or redress poor quality in, existing services. Within the area of intellectual disabilities, the development of the normalisation principles across the Scandinavian countries following World War II was derived from parent initiatives. Then in the late 1960s and early 1970s, Nirje and Wolfensberger working on behalf of parents subsequently developed the notion of social role valorisation (Wolfensberger, 1972; Wolfensberger, 1983/2011); this being a powerful example of how parents challenge and transform service paradigms. People with intellectual disabilities and high support needs often rely on others who know them well to 
advocate on their behalf, and parents who have the best interests of their child in mind can be powerful agents in transforming services and service delivery for people with intellectual disabilities.

\section{Stigmatisation of intellectual disabilities}

Both the focus group and case study illustrated that there is profound stigma attached to intellectual disabilities in Lao PDR. It was also suggested during discussion that some families may not access services to avoid association with intellectual disabilities, particularly families with high social status, illustrating the impact of stigma. This is consistent with other research across the region with Komardjaja (2005) suggesting that, while people with disabilities and their families are stigmatised in Indonesia, people with intellectual disabilities are the most stigmatised. Negative attitudes and perceptions have contributed to a lack of services and funding to assist people.

It is probable that the stigma attached to intellectual disabilities in Lao PDR has similar origins to that identified in other countries in the region. In many Asian countries, it is expected that children will care for their parents in old age. It has been proposed that this cultural expectation has influenced negative attitudes towards having a child with disabilities, as parents will always be the caregivers, even later in life when they may need care themselves (Shobana and Saravanan, 2014). This cultural expectation is somewhat different than in Western countries, where older people receive welfare benefits, and so it is proposed that this partly accounts for more positive attitudes towards disabilities in the Western world (Shobana and Saravanan, 2014). Another factor influencing negative attitudes towards children with intellectual disabilities in Asia is a lack of knowledge about disabilities, for example, beliefs that it is contagious or is the result of sin or karma, causing parents to be ashamed to be seen with their children (Shobana and Saravanan, 2014). 


\section{Limitations}

While this article draws on a comprehensive mixed-methods study, there are a number of limitations. First, the screening survey is representative of the survey sites, but not Lao PDR more broadly. Second, while this is one of a few attempts to provide an indication of the prevalence of intellectual disabilities in Lao PDR, the efficacy of the Washington Group questions to capture intellectual and other developmental disabilities is poor (Loeb, 2013). While difficulties remembering or concentrating can indicate intellectual disabilities, these difficulties can also indicate psychosocial disabilities, acquired brain injuries, and reduced cognitive functioning associated with ageing, such as dementia. Further, difficulties remembering or concentrating are not the only manifestations of intellectual disabilities. Thus, the prevalence of intellectual disabilities for Lao PDR presented in this article should only be viewed as a general estimate. Third, as is often the case when adopting qualitative research approaches, neither the intellectual disabilities focus group nor the case study of AfA included any form of randomisation. Rather, participants were purposively selected to represent a broad spectrum of stakeholders based on their depth of experiences, expertise, and roles. Finally, much of the research was conducted in the Lao language with the aid of translators and interpreters. No doubt subtleties are likely to have been lost. As well, the very different characteristics of English and Laotian mean that conversations and terminology about intellectual disabilities will not always translate well.

\section{Conclusion}

In providing an overview of intellectual disabilities in Lao PDR, this may well be the first comprehensive snapshot of intellectual disabilities for the country offered in the formal literature. It presents information around prevalence as well as key stakeholders' perspectives around services for people with intellectual disabilities and thus fills an important gap. 
The case study illustrates the crucial role the AfA has played, as a parent association, in developing services for their children with autism and intellectual disabilities. Historically, parents and other family members have played, and continue to play, seminal roles in improving services and quality of life for persons with disabilities generally and intellectual disabilities specifically across the world. The study has also identified substantial stigmatisation of people with disabilities in Lao PDR. This has also been documented in other countries, and the source of stigma for people with intellectual and other developmental disabilities and their families may indeed have specific cultural or religious origins across the region. Redressing this is also crucial for effective service delivery. Improving outcomes for people with intellectual disabilities in any country cannot solely rely on leapfrogging based on innovative models and practices developed elsewhere which place emphasis on social inclusion and community integration. However, much has been done to improve social and economic access to health, education and employment services for people with disabilities in Lao PDR and, in time, people with intellectual disabilities will begin to benefit from these advances. 


\section{Reference}

AlHW. (2008) Disability in Australia: intellectual disability. AlHW bullitin no.67. Cat. no. AUS110., Canberra: Australian Government.

Amar-Singh H. (2008) Meeting the needs of children with disability in Malaysia. Medical Journal of Malaysia 63: 1-3.

Australian Government. (2008) Development for all: Towards a disability-inclusive Australian aid program 2009-2014, Canberra: Australian Agency for International Development (AusAID).

Chou YC, Lee YC, Lin LC, et al. (2008) Social services utilization by adults with intellectual disabilities and their families. Social Science and Medicine 66: 2474-2485.

Cocks E, Thoresen SH, O'Brien P, et al. (2016) Examples of individual supported living for adults with intellectual disability. Journal of Intellectual Disabilities 20: 100-108.

Cohen L, Brown R and McVilly K. (2015) The World Report on Disability: challenges to application and translation for individuals with intellectual and developmental disabilities. Journal of Policy and Practice in Intellectual Disabilities 12: 77-78.

Commonwealth of Australia. (2015) Development for All 2015-2020: Strategy for strengthening disability-inclusive development in Australia's aid program, Barton, ACT: DFAT.

Cooper R. (2014) Laos: Work in Progress, Laos: Lao Insight Books.

Deng M and Holdsworth JC. (2007) From unconscious to conscious inclusion: meeting special education needs in West China. Disability \& Society 22: 507-522.

Durham J, Hill PS and Hoy D. (2013) The underreporting of landmine and explosive remnants of war injuries in Cambodia, the Lao People's Democratic Republic and Viet Nam. Bulletin of the World Health Organization 91: 234-236.

Durham J and Hoy D. (2013) Burden of injury from explosive remnants of conflict in Lao PDR and Cambodia. Asia-Pacific Journal of Public Health 25: 124-133. 
Einfeld S, Stancliffe R, Gray K, et al. (2012) Interventions provided by parents for children with intellectual disabilities in low and middle income countries. Journal of Applied Research in Intellectual Disabilities 25: 135-142.

Elsabbagh M, Divan G, Koh Y-J, et al. (2012) Global prevalence of autism and other pervasive developmental disorders. Autism Research 5: 160-179.

Emerson E. (2007) Poverty and people with intellectual disabilities. Mental Retardation and Developmental Disabilities Research Reviews 13: 107-113.

Grimes P. (2009) A quality education for all: The history of the Lao PDR Inclusive Education Project 1993-2009, Vientiane: Save the Children.

Grimes P, Sayarath K and Outhaithany S. (2011) The Lao PDR Inclusive Education Project 1993-2009: Reflections on the impact of a national project aiming to support the inclusion of disabled students. International Journal of Inclusive Education 15: 1135-1152.

Hall AC and Kramer J. (2009) Social capital through workplace connections: Opportunities for workers with intellectual disabilities. Journal of Social Work in Disability \& Rehabilitation 8: 146-170.

Harris J. (2006) Intellectual Disability: Understanding its Development, Causes, Classification, Evaluation and Treatment, New York: Oxford University Press.

Jacobs B, Ir P, Annear P, et al. (2012) Addressing access barriers to health services: an analytical framework for selecting appropriate interventions in low-income Asian countries. Health Policy and Planning: 27:288-300

Juneja M, Jain R, Singhal S, et al. (2012a) Availing services for developmental disabilities: parental experiences from a referral center in developing country. Indian Journal of Pediatrics 79: $1213-1217$.

Juneja M, Mukherjee SB, Sharma S, et al. (2012b) Evaluation of a parent-based behavioral intervention program for children with autism in a low-resource setting. Journal of Pediatric Neurosciences 7: 16-18. 
Komardjaja I. (2005) The place of people with intellectual disabilities in Bandung, Indonesia. Health and Place 11: 117-120.

Kuipers P and Maratmo J. (2011) A low-intensity approach to early intervention and detection of childhood disability in Central Java: long-term findings and implications for "inclusive development". Disability, CBR and Inclusive Development 22: 3-14.

Kwok HW and Chui EM. (2008) A survey on mental health care for adults with intellectual disabilities in Asia. Journal of Intellectual Disability Research 52: 996-1002.

Lao People's Democratic Republic. (2014) Decree on Persons with Disabilities. Vientiane Capital: Government of the Lao PDR.

Lao Statistics Bureau. (2015) Lao population and housing census 2015: Provisional report, Vientiane Capital: Ministry of Planning and Investment.

Loeb M. (2012) A White Paper on Disability Measurement. Disability and International Development 23: 4-11.

Loeb M. (2013) Disability statistics: an integral but missing (and misunderstood) component of development work. Nordic Journal of Human Rights 31: 306-324.

Mandans JH, Loeb ME and Altman BM. (2011) Measuring disability and monitoring the UN Convention on the Rights of Persons with Disabilities: the work of the Washington Group on Disability Statistics. BMC Public Health 11: S4.

Maulik P, Mascarenhas C, Mathers C, et al. (2011) Prevalence of intellectual disability: a metaanalysis of population-based studies. Research in Intellectual Disabilities 32: 419-436.

Messerli P, Heinimann A, Epprecht M, et al. (2008) Socio-economic atlas of the Lao PDR an analysis based on the 2005 Population and Housing Census. Swiss National Center of Competence in Research (NCCR) North-South, University of Bern, Bern and Vientiane: Geographica Bernensia.

Miles MB and Huberman AM. (1994) Qualitative data analysis, Thousand Oaks, London, and New Delhi: Sage Publications. 
Mitra S, Posarac A and Vick B. (2011) Disability and poverty in developing countries: A snapshot from the World Health Survey. SP Discussion Paper No. 1109: The World Bank.

Mont D. (2007) Measuring disability prevalence. SP Discussion Paper NO. 0706. The World Bank.

Morikawa M, Taylor S and Persons M. (1998) Death and injuries due to unexploded ordnance (UXO) in northern Lao PDR (Laos). Injury 29: 301-304.

National Statistical Office and Ministry of Information and Communication Technology. (2014) The 2012 disability survey. Available at:

\section{http://web.nso.go.th/en/survey/disabi/data/2012 Full\%20Report.pdf. Accessed 2} November 2016.

Nazri NA and Ismail Z. (2015) Educational building facilities for children with autism in Malaysia. Jurnal Teknologi 78: 49-54.

Peters B and Forlin C. (2011) Chinese children with ASD in Hong Kong (SAR): Development of inclusive practice. Journal of Research in Special Educational Needs 11: 87-98.

Poon K. (2015) Context, service provision, and reflections on future directions of support for individuals with intellectual disability in Singapore. Journal of Policy and Practice in Intellectual Disabilities 12: 100-107.

Robertson J, Emerson E, Hatton C, et al. (2012) Efficacy of community-based rehabilitation for children with or at significant risk of intellectual disabilities in low-and middle-income countries: a review. Journal of Applied Research in Intellectual Disabilities 25: 143-154.

Scior K, Hamid A, Hastings R, et al. (2015) Intellectual disabilities: Raising awareness and combating stigma - A global review. London: University College London.

Shobana M and Saravanan C. (2014) Comparative study on attitudes and psychological problems of mothers towards their children with developmental disability. East Asian Archives of Psychiatry 24: 16-22. 
Steering Committee for Census of Population and Housing. (2004) Questionnaire used for Census of Population and Housing 2005, English version. Available at: http://catalog.ihsn.org/index.php/catalog/3149/download/46457. Accessed 30 May 2016. Thomas P. (2005) Disability, poverty and the Millennium Development Goals: Relevance, challenges and opportunities for DFID, Ithaca, New York: Cornell University ILR School.

Thoresen SH, Fielding A, Gillieatt S, et al. (2014) Facilitating disability inclusive development in Lao PDR by improving access to social and economic services: a scoping review of contexts and concepts. Ratchasuda International Conference on Disability. Bangkok, Thailand. Available at http://link.library.curtin.edu.au/p?cur digitool dc201006. Accessed 6 April 2017.

UNFPA. (2011) People with disabilities in Viet Nam: Key findings from the 2009 Viet Nam population and housing census. Ha Noi: United Nationas Population Fund.

United Nations. (2015) Transforming our world: The 2030 Agenda for Sustainable Development. United Nations. Available at: https://sustainabledevelopment.un.org/post2015/transformingourworld/publication. Accessed 25 June, 2016.

United Nations. (2016) List of Least Developed Countries (as of May 2016). Available at: http://www.un.org/en/development/desa/policy/cdp/ldc/ldc list.pdf. Accessed 17 May 2016.

United Nations Development Programme. (2012) The Lao development journey towards graduation from $L D C$ status. Available at:

http://www.la.undp.org/content/dam/laopdr/docs/Reports\%20and\%20publications/UNDP LA LDC Pamphlet 2012.pdf. Accessed 17 May 2016.

United Nations Economic and Social Commission for Asia and the Pacific. (2012) Incheon Strategy to "make the right real" for persons with disabilities in Asia and the Pacific, Bangkok: United Nations Publications. 
United Nations Economic and Social Commission for Asia and the Pacific. (2014) ESCAP guide on disability indicators for the Incheon Strategy, Bangkok: United Nations Publications.

United Nations General Assembly. (2006) Convention on the rights of persons with disabilities.

Available at: http://www.un.org/disabilities/convention/conventionfull.shtml. Accessed 18 November 2009.

Washington Group. (2014) Module on child functioning and disability: Washington Group on

Disability Statistics / UNICEF. Available at:

http://www.cdc.gov/nchs/data/washington group/meeting13/wg13 unicef child disability background.pdf. Accessed 25 June 2016.

Wolfensberger W. (1972) The principle of normalization in human services, Toronto: National Institute on Mental Retardation.

Wolfensberger W. (1983/2011) Social role valorization: A proposed new term for the principle of normalization. Intellectual and Developmental Disabilities 49: 435-440.

World Health Organization. (2001) International classification of functioning, disability and health (ICF), Geneva: World Health Organization.

World Health Organization. (2007). Atlas: global resources for persons with intellectual disabilities. Geneva: WHO.

World Health Organization and The World Bank. (2011) World report on disability, Malta: World Health Organization. 